\title{
Aedes Aegypti. Estudo Piloto sobre a Efetividade de Deltametrina e Lambda-Cialotrina em Culicídeos com Capacidade Vetorial
}

\author{
Camille Zambiasi Medeiros ${ }^{\prime}$ :Letícia Carolina da Cruz'; Alexandre Ehrhardt ${ }^{l}$
}

$\bowtie \underline{\text { camillezambiasimedeiros@hotmail.com }}$

1. Universidade Luterana do Brasil.

Histórico do Artigo:

Resumo: 0 Aedes aegyptié o mosquito vetor de maior estima para a saúde pública, sendo o transmissor de inúmeras doenças endêmicas no mundo. Os programas de controle vetorial utilizam como principais inseticidas químico, os organofosforados e piretróides, que auxiliam contra a proliferação de criadouros de mosquitos. Porém, alguns fatores de resistência a inseticidas do Aedes dificultam a vigilância epidemiológica e monitoramento. Com isso objetivou-se avaliar a efetividade de um produto com ação larvicida, a partir dos dois compostos: Deltametrina 0,75\% + Lambda-cialotrina 0,25\%, em larvas expostas ao meio ambiente, locais com supostos focos na cidade de Carazinho, Rio Grande do Sul. As larvas foram coletadas em criadouros e armazenadas em recipientes até serem levadas ao laboratório para análise. Para todos os experimentos, foi utilizada uma diluição padrão indicada de uso, sendo rediluída nas proporções de: $1.000 \mu \mathrm{L}, 500 \mu \mathrm{L}, 200 \mu \mathrm{L}, 100 \mu \mathrm{L}, 50 \mu \mathrm{L}$ e $25 \mu \mathrm{L}$ em $100 \mathrm{~mL}$ de $\mathrm{H}_{2} 0$. Na avaliação das diluições testadas, foi possível observar um padrão de inibição com intervalo de tempo máximo de 30 minutos, havendo $100 \%$ de mortalidade, indicando alto nível susceptibilidade ao produto em experimentação. Ao serem avaliadas as larvas de culicídeos selvagens expostas a aplicação do produto com ação larvicida na combinação dos piretróides DMT e LCT, o mesmo alcançou a efetividade desejada.

Palavras-chave: Vetores artrópodes, Dengue, Saúde Pública, Larvicidas.

\section{Aedes Aegypti. Pilot Study on the Effectiveness of Deltametrine and Lambda- Cyalotrin in Culicides With Vectorial Capacity}

\begin{abstract}
Aedes aegypti is the vector mosquito of most esteem for public health, being the transmitter of numerous endemic diseases in the world. Vector control programs use organophosphates and pyrethroids as the main chemical insecticide, which help against the proliferation of mosquitoes. However, some resistance factors of Aedes, demonstrate greater difficulty for surveillance and monitoring. Thus, the objective was to evaluate the effectiveness of a product with larvicidal action, using the compounds $0.75 \%$ Deltamethrin $+0.75 \%$ Lambdacyhalothrin, in larvae exposed to the environment, in neighborhoods with a focus on the city of Carazinho, Rio Grande do Sul. The larvae were collected in breeding sites, and stored in containers until they were taken to the laboratory for analysis. For all experiments, a standard dilution indicated for use was used, being diluted in the proportions of: 1,000 $\mathrm{L}, 500 \mu \mathrm{L}, 200 \mu \mathrm{L}, 100 \mu \mathrm{L}, 50 \mu \mathrm{L}$ and $25 \mu \mathrm{L}$ in $100 \mathrm{~mL}$ of H20. In the evaluation of the tested dilutions, it was possible to observe an inhibition pattern with a maximum time interval of 30 minutes, with $100 \%$ mortality, indicating a high level of susceptibility to the product under experiment. When evaluating the larvae of wild culicids exposed to the application of the product with larvicidal action in the combination of the pyrethroids DMT and LCT, it achieved the desired effectiveness.
\end{abstract}

Keywords: Arthropod vectors, Dengue, Public health, Larvicides. 


\section{Aedes Aegypti: Estudio Piloto sobre la Eficacia de Deltametrine y Lambda- Cyalotrin en Culicidas con Capacidad Vectorial}

Resumen: Aedes aegypti es el mosquito vector más estimado para la salud pública, y es el transmisor de numerosas enfermedades endémicas en el mundo. Los programas de control de vectores utilizan organofosforados y piretroides como el principal insecticida químico, que ayuda contra la proliferación de los sitios de reproducción. Sin embargo, algunos factores de resistencia del Aedes demuestran una mayor dificultad para la vigilancia y el monitoreo. Por lo tanto, el objetivo fue evaluar la efectividad de un producto con acción larvicida, utilizando los compuestos $0.75 \%$ Deltametrina + $0.75 \%$ Lambda-cihalotrina, en larvas expuestas al medio ambiente, en vecindarios con un enfoque en la ciudad de Carazinho, Río Grande do Sul. Las larvas fueron recolectadas en lugares con supuestos sitios de reproducción y almacenadas en contenedores hasta que fueron llevadas al laboratorio para su análisis. Para todos los experimentos, se usó una dilución estándar indicada para su uso, que se diluyó en las proporciones de: 1,000 $\mu \mathrm{L}, 500 \mu \mathrm{L}, 200 \mu \mathrm{L}, 100 \mu \mathrm{L}, 50 \mu \mathrm{L}$ y $25 \mu \mathrm{L}$ en $100 \mathrm{~mL}$ de H20. En la evaluación de las diluciones probadas, fue posible observar un patrón de inhibición con un intervalo de tiempo máximo de 30 minutos, con una mortalidad del $100 \%$, lo que indica un alto nivel de letalidad del producto bajo experimento. Al evaluar las larvas de culicidas silvestres expuestas a la aplicación del producto con acción larvicida en la combinación de los piretroides DMT y LCT, se logró la efectividad deseada.

Palabras clave: Vectores de artrópodos, Dengue, Salud pública, Larvicidas.

\section{INTRODUÇ̃̃o}

0 Aedes aegypti é um mosquito de origem africana de fácil adaptação selvagem e doméstica, que adquiriu uma constante migração a diferentes países juntamente ao homem. Esse vetor introduziu-se possivelmente nas Américas durante as primeiras colonizações europeias a bordo de embarcações vindas da Europa, cruzando o Atlântico (BRAGA; VALLE, 2007).

Segundo Manual de Normas Técnicas sobre o manejo da Dengue da FUNASA (BRASIL, 2001), o Ae. aegypti pertence ao ramo Arthropoda, classe Hexapoda, ordem Diptera, família Culicidae do gênero Aedes, caracterizado como o principal vetor da dengue. Seu desenvolvimento ocorre através de metamorfose completa, possuindo um ciclo com quatro fases, sendo elas: ovo, larva, pupa e adultos.

Somente as fêmeas são hematófagas, sendo a alimentação sanguínea feita através da picada preferencialmente em humanos onde utilizam a albumina do sangue como alimento. Já, os machos alimentam-se de substâncias adocicadas, como néctar e seiva de plantas (BRAGA; VALLE 2007 p. 280; STEFANI et al., 2009).

De fácil adaptação, o mosquito se acomoda em ambientes urbanos ou rurais, ocupando recipientes, como tanques de armazenamento de água, vasos de plantas, calhas, entre outros, chamados de criadouros. É uma espécie antropofílica, apresentando hábitos diurnos, onde se alimenta e deposita seus ovos ao amanhecer e no período vespertino em ambientes adequados para o crescimento (STEFANI et al., 2009). 
Aedes Aegypti: Estudo Piloto sobre a Efetividade de Deltametrina e Lambda-Cialotrina em Culicídeos com Capacidade Vetorial

A reprodução do A. aegypti ocorre através do acasalamento nos primeiros dias da fase adulta na qual as fêmeas armazenam o esperma, e durante esse período precisam realizar a hematofagia, condição importante para formação os ovos. A fêmea pode desovar até 1.500 mosquitos durante a sua vida, sendo espalhados a vários criadouros. Durante esse processo pode ocorrer a transmissão vertical na qual a fêmea infectada com o vírus da dengue por exemplo, pode passar para as larvas filhas, e quando adultas, já serem capazes de transmitirem o vírus para o homem (P0NTE et al., 2011).

A dengue é uma doença causada por um arbovírus pertencente ao gênero Flavivirus e à família Flaviridae ligada a quatro subtipos antigenicamente distintos do vírus sendo eles DENV1, DENV-2, DENV-3 e DENV-4. Ocorre em áreas de clima tropical e subtropical, sendo uma das principais doenças presentes nessas localidades, tendo como principal vetor o mosquito A. aegypti. (GUZMAN; HARRIS, 2014; P0NTE et al., 2011).

As alternativas utilizadas para o controle da dengue no Brasil atualmente abrangem métodos químicos, físicos, biológicos e genéticos, cada um apresentando particularidades, vantagens e desvantagens (CASTR0; QUEIR0Z, 2011).

Os programas de controle do A. aegypti, utilizam como principais inseticidas químicos os organofosforados e piretróides, que auxiliam contra a proliferação de mosquitos. Porém, alguns fatores de resistência do Aedes demonstram maior dificuldade para o controle dos mesmos, sendo que a resistência adquirida pelo mosquito é decorrente do uso desses inseticidas de primeira escolha (DINIZ et al., 2014).

Devido a ocorrência de resistência em populações de A. aegypti aos inseticidas em uso, o PNCD (Programa Nacional de Controle da Dengue), vem gradualmente promovendo a substituição dos mesmos. 0s organofosforados (malationa, fenitrotiona e temefós) foram os primeiros a substituir os organoclorados. Por sua vez, os piretróides vem sendo usados como alternativa para substituição dos organofosforados, devido a sua alta eficiência contra mosquitos adultos e as baixas concentrações necessária do produto ativo (ZARA et al., 2016).

0 uso de larvicidas é uma das ferramentas mais utilizada contra a proliferação do mosquito, considerando como critérios para eficácia, a baixa toxicidade aos mamíferos e o baixo impacto ambiental, apresentando atividade contra as espécies alvo e proporciona, um efeito de longa duração e uma redução da aplicação do produto (INVEST; LUCAS, 2008).

Há mais de quinze anos o uso de inseticidas químicos tem sido uma estratégia adotada para controle de vetores. Dentre estes, os organofosforados são amplamente utilizados, aplicados em volume ultrabaixo como adulticida durante períodos endêmicos ou como larvicida 
aplicado em recipientes com água em locais com potencial de reprodução de mosquitos vetores (ROLDAN et al., 2013).

Com a resistência ao DDT, houve a procura de outros recursos e ações, como o aumento da dose aplicada e o desenvolvimento de novas moléculas, procedendo na série de diferentes classes de moléculas tóxicas, como o uso de outros organoclorados, organofosforados, carbonatos e piretróides, sendo todos não seletivos e capazes de levar a resistência aos insetos (MELÓ, 2009).

Dentre os organofosforados empregados para o controle do A. aegypti, o teméfos foi utilizado extensivamente em vários países contra vetores biológicos, adentrado no mercado em 1965 pela American Cyanamid Company, tendo formulações conhecidas como Abate, Difos, Biothion, Abathion, Nimitex e Swebate. Foi usado inicialmente no controle de mosquitos do gênero Simulium e outros insetos na fase larval aquática. No Brasil, foi utilizado entre os anos de 1967 a 1998, no controle do vetor A aegypti, sendo na época o pesticida mais utilizado (MÉL0, 2009).

A Deltametrina e a Lambda-cialotrina fazem parte do grupo dos piretróides, classificadas como inseticida, formicida e acaricida, tendo a classificação toxicológica classe III, indicadas e autorizadas para uso agrícola. 0 princípio ativo da Deltametrina segue a concentração máxima permitida de $10 \%$ p/p para entidades especializadas e campanhas públicas em forma líquida ou pastosa. A Lambda-cialotrina em suspenção concentrada segue a concentração de 10,6\% p/v para entidades especializadas e 2,5 p/v para emulsão em água em campanhas públicas (BRASIL, 2003).

Os piretróides atuam com um efeito expressivo sobre os canais de sódio neural, intervindo em seu fechamento através da ação de prolongamento da permeabilidade dos canais dos íons de $\mathrm{Na}^{+}$para o interior das células, suprimindo o efluxo de potássio. Nos mamíferos há vários canais de sódio de formato semelhante que variam suas propriedades biofísicas e farmacológicas, podendo levar a alguma sensibilidade aos piretróides (SANTOS; AREAS; REYES, 2007).

Até o mês de setembro de 2017, foram notificados 219.040 casos prováveis de dengue em todo o país, tendo uma redução de 84,8\% em relação ao mesmo período de 2016 (1.442.208). Com relação ao número de óbitos, também houve queda significativa (87\%), reduzindo de 678 óbitos em 2016 para 88 em 2017. Da mesma forma, os registros de dengue grave e com sinais de alarme tiveram queda de 79,2\% e 77,7\%, respectivamente, de um ano para outro (BRASIL, 2017). 
Aedes Aegypti: Estudo Piloto sobre a Efetividade de Deltametrina e Lambda-Cialotrina em Culicídeos com Capacidade Vetorial

Tendo em vista todo o exposto apresentado e a importância em relação a prevenção da disseminação da dengue e a resistência de populações de Aedes, o presente trabalho teve como objetivo avaliar a ação larvicida de Deltametrina e Lambda-cialotrina, visando uma sinestesia dos compostos escolhidos, para uma maior efetividade do produto em menores concentrações, diante a proliferação do mosquito A. aegypti e outros culicídeos.

\section{MATERIAL E MÉTODOS}

A presente pesquisa foi realizada através de um estudo exploratório descritivo com abordagem comparativa de mecanismos de ação e de caráter experimental. Foram considerados como critérios de inclusão todas as larvas e pupas vivas de mosquitos presentes no meio ambiente urbano/domiciliar, sendo de localidades com suspeita de criadouros do mosquito $A$. aegypti, e não sendo tratadas com nenhum outro inseticida/larvicida, segundo mapa urbano da cidade.

As coletas aconteceram entre os meses de fevereiro e julho de 2017, com o auxílio da Vigilância Ambiental em Saúde do município de Carazinho-RS para as coletas a campo.

As possíveis larvas do gênero Aedes, durante a coleta a campo, apresentaram fotofobia visível, com movimentos desordenados e mais ligeiros, além de expressarem diante a luz do sol, um brilho translucido, diferente de algumas outras espécies que apresentaram cores mais enegrecidas diante a comparação.

Seguindo a mesma linha de experimento segundo, Coelho et al. (2017), a constatação da mortalidade de $100 \%$ das larvas, foi após observação de sua absoluta imobilização ao fundo dos recipientes em ensaio, evidenciando a letalidade do produto testado.

\section{Diluição do produto concentrado}

Composição inicial: Deltametrina (DMT) 0,75\% + Lambda-cialotrina (LCT) 0,25\%.

A medida da diluição sugerida inicialmente em testes experimentais para validação do uso comercial segundo fornecedor é $50 \mathrm{~mL}$ do produto concentrado para $4.000 \mathrm{~mL}$ de água (diluição 1/80). Para todos os experimentos em laboratório foi utilizada a diluição padrão para soluções estoque de $10 \mathrm{~mL}$ do produto para $800 \mathrm{~mL}$ de água deionizada.

Foram realizadas oito coletas de campo, nos bairros Oriental, Cantares e Conceição da cidade de Carazinho-RS. As larvas e pupas foram coletadas com o auxílio de pipetas plásticas e 
armazenadas em recipientes de vidro ou plástico com água do próprio criadouro, até serem levadas ao laboratório para análise. As análises foram realizadas no Laboratório Escola da ULBRA Campus Carazinho, no mesmo tempo que as coletas eram obtidas, sendo as mesmas processadas e descartadas seguindo as normas e políticas do Laboratório.

Todos os experimentos foram realizados em copos de béquer esterilizados em calor úmido com capacidade de $250 \mathrm{~mL}$, sendo acrescidos para diluição inicial, $100 \mathrm{~mL}$ de água deionizada, seguidas das diferentes concentrações propostas do produto em estudo.

Primeiro experimento: Para todos os experimentos foi padronizado um tempo de observação diante as amostras de 30 a 45 minutos.

$1^{\mathrm{a}}$ aplicação: foram acrescidos $100 \mathrm{~mL}$ de água deionizada para $1 \mathrm{~mL}$ da diluição padrão em dois copos de béquer, sendo submetidas 5 larvas e 5 pupas, apresentando a diluição final $1 / 800$.

2a aplicação: concentração de $100 \mathrm{~mL}$ de água deionizada para $500 \mu \mathrm{L}$ da diluição padrão, sendo submetidas 6 larvas e 6 pupas, apresentando diluição final 1/1600.

Segundo experimento: As amostras desse experimento foram coletadas pelo Departamento de Vigilância Ambiental em Saúde do município, dois dias após as primeiras aplicações, sendo atribuída a diluição padrão (em 100 mL de água deionizada), para outras menores diluições, testadas repetidamente durante 0 estudo, sendo elas: $50 \mu \mathrm{L}$ da diluição padrão (diluição final de 1/16000) na qual foram submetidas ao teste 4 larvas e 1 pupa; $100 \mu \mathrm{L}$ da diluição padrão (diluição final de 1/8000) na qual foram submetidas ao teste 4 larvas, e 200 $\mu \mathrm{L}$ da diluição padrão (diluição final de 1/4000) na qual foram submetidas ao teste 4 larvas.

Terceiro experimento: Esse processo iniciou-se com caráter semelhante ao anterior, sendo as amostras obtidas pelo Departamento de Vigilância Ambiental em Saúde do município, após 18 dias do segundo experimento, sendo as diluições anteriores para $(100 \mu \mathrm{L}, 50 \mu \mathrm{L})$ repetidas com a finalidade de confirmar a eficácia e o tempo de sensibilização para cada aplicação. Além dessa repetição, foi manipulada uma menor concentração, $25 \mu \mathrm{L}$ da diluição padrão (diluição final de 1/32000), sendo submetida 3 larvas para cada diluição respectivamente. 
Aedes Aegypti: Estudo Piloto sobre a Efetividade de Deltametrina e Lambda-Cialotrina em Culicídeos com Capacidade Vetorial

Quarto experimento: 38 amostras larvárias foram obtidas de um possível foco após quinze dias do terceiro experimento, com a finalidade de manter os ciclos para desenvolvimento da fase alado do mosquito. Esse processo levou à espera de mais quinze dias para observação final. Das amostras não desenvolvidas (todas), ou seja, estacionadas ao ciclo larvário, foi realizado a repetição das duas menores concentrações diante a diluição padrão estabelecida, para aproveitamento das mesmas, sendo nas aplicações:

$1^{\mathrm{a}}$ aplicação: $25 \mu \mathrm{L}$ da diluição padrão, sendo submetidas 20 larvas.

$2^{\mathrm{a}}$ aplicação: $50 \mu \mathrm{L}$ da diluição padrão, sendo submetidas 18 larvas.

Quinto experimento: Após 106 dias do último experimento, uma nova coleta foi realizada, com a finalidade de analisar: as populações encontradas, a estabilidade do princípio ativo e a repetição das diluições já testadas. 35 larvas foram capturadas e submetidas as concentrações conhecidas e analisadas ao tempo de sensibilização de cada uma delas.

$1^{\mathrm{a}}$ aplicação: $25 \mu \mathrm{L}$ da diluição padrão, sendo submetidas 8 larvas.

$2^{\mathrm{a}}, 3^{\mathrm{a}} \mathrm{e} 4^{\mathrm{a}}$ aplicação: 9 larvas submetidas para cada concentração da diluição padrão (500 $\mu \mathrm{L}, 200 \mu \mathrm{L}, 50 \mu \mathrm{L}$ respectivamente).

\section{RESULTAD0S E DISCUSSÃ0}

Na avaliação das amostras obtidas da coleta a campo, foi possível observar um padrão de inibição diante a concentração da DMT $(0,75 \%)+$ LCT $(0,25 \%)$, com intervalos de tempo aproximados nas diferentes diluições exibidas. Todos os experimentos realizados em laboratório seguiram um período de observação, sendo possível determinar o tempo estimado para cada diluição proposta.

As larvas submetidas as dosagens de $25 \mu \mathrm{L}$ e $50 \mu \mathrm{L}$ da diluição inicial do produto em 100 mL de água, foram observadas até inibição total da motilidade, por volta de vinte minutos, apresentando 100\% de mortalidade. As larvas testadas nas diluições $100 \mu \mathrm{L}$ e $200 \mu \mathrm{L}$ levaram 0 tempo de dezesseis minutos para inibir 98\%. As larvas testadas na diluição de $500 \mu \mathrm{L}$ responderam ao produto após doze minutos submetidas, apresentando 98\% de inibição da motilidade. Sendo assim, todas as larvas testadas nas diluições anteriores apresentaram após trinta minutos de observação, $100 \%$ de inibição da motilidade, evidenciando alto nível de susceptibilidade ao produto em experimentação, durante esse tempo descrito. 
Tabela 1. Resultados dos experimentos realizados, apresentando a inibição da motilidade das larvas expostas ao produto.

\begin{tabular}{cccccccc}
\hline $\begin{array}{c}\text { Dosagens } \\
\text { seguidas da } \\
\text { diluição } \\
\text { padrão }(\mu \mathrm{L}) \\
1 / 80\end{array}$ & $\begin{array}{c}\mathbf{1}^{\mathbf{0}} \\
\text { Experimento } \\
\text { Larvas }(\boldsymbol{n})\end{array}$ & $\begin{array}{c}2^{\mathbf{0}} \\
\text { Experimento } \\
\text { Larvas }(\boldsymbol{n})\end{array}$ & $\begin{array}{c}3^{\mathbf{0}} \\
\text { Experimento } \\
\text { Larvas }(\boldsymbol{n})\end{array}$ & $\begin{array}{c}4^{\mathbf{0}} \\
\text { Experimento } \\
\text { Larvas }(\boldsymbol{n})\end{array}$ & $\begin{array}{c}5^{\mathbf{0}} \\
\text { Experimento } \\
\text { Larvas }(\boldsymbol{n})\end{array}$ & $\begin{array}{c}\text { Inibição da } \\
\text { motilidade } \\
(\boldsymbol{n})\end{array}$ & $\begin{array}{c}\text { Letalidade } \\
(\%)\end{array}$ \\
\hline $1.000 \mu \mathrm{L}$ & 5 & 0 & 0 & 0 & 0 & 5 & $100 \%$ \\
$500 \mu \mathrm{L}$ & 6 & 0 & 0 & 0 & 0 & 6 & $100 \%$ \\
$200 \mu \mathrm{L}$ & 0 & 4 & 0 & 0 & 9 & 13 & $100 \%$ \\
$100 \mu \mathrm{L}$ & 0 & 4 & 3 & 0 & 9 & 16 & $100 \%$ \\
$50 \mathrm{Ml}$ & 0 & 4 & 3 & 18 & 9 & 34 & $100 \%$ \\
$25 \mu \mathrm{L}$ & 0 & 0 & 3 & 20 & 8 & 31 & $100 \%$ \\
Total: & 11 & 12 & 9 & 38 & 35 & 105 & $100 \%$ \\
\hline
\end{tabular}

Fonte: Medeiros C. Z.; Ehrhardt A; Cruz L.C. 2017.

Na Tabela 1 encontram-se os resultados obtidos em todos os experimentos realizados com larvas durante o período de fevereiro a julho de 2017, apresentando as dosagens utilizadas, seguidas do número de larvas submetidas em cada experimento e o número de larvas inibidas, expondo assim, a letalidade do produto em experimentação.

Tabela 2. Resultados dos experimentos realizados, apresentando a inibição da motilidade das pupas expostas ao produto.

\begin{tabular}{cccccc}
\hline $\begin{array}{c}\text { Dosagens seguidas } \\
\text { da diluição padrão } \\
(\mu \mathrm{L}) 1 / 80\end{array}$ & Diluições & \multicolumn{4}{c}{ Larvas } \\
& & 0 & 0 & 5 & $100 \%$ \\
\hline $1.000 \mu \mathrm{L}$ & $1 / 800$ & 0 & 0 & 6 & $100 \%$ \\
$500 \mu \mathrm{L}$ & $1 / 1600$ & 0 & 13 & 0 & $98 \%$ \\
$200 \mu \mathrm{L}$ & $1 / 4000$ & 0 & 16 & 0 & $98 \%$ \\
$100 \mu \mathrm{L}$ & $1 / 8000$ & 34 & 0 & 0 & $100 \%$ \\
$50 \mu \mathrm{L}$ & $1 / 16000$ & 31 & 0 & 0 & $100 \%$ \\
$25 \mu \mathrm{L}$ & $1 / 32000$ & & & & \\
\hline
\end{tabular}

Fonte: Medeiros C. Z.; Ehrhardt A; Cruz L.C. 2017. 
Aedes Aegypti: Estudo Piloto sobre a Efetividade de Deltametrina e Lambda-Cialotrina em Culicídeos com Capacidade Vetorial

Na tabela 2, a efetividade do produto foi apresentada na fase pupal de culicídeos, tento a letalidade do produto em experimentação assegurada também a essa fase.

Tabela 3. Mortalidade das larvas expostas e eficiência (E\%) do produto em experimento por minuto (min).

\begin{tabular}{cccccc}
\hline $\begin{array}{c}\text { Dosagens seguidas } \\
\text { da diluição padrão } \\
(\mu \mathrm{L}) 1 / 80\end{array}$ & Diluições & \multicolumn{5}{c}{$\begin{array}{c}\text { Lorvas } \\
20 \text { min } 16 \text { min } 12 \mathrm{~min}\end{array}$} & Total \\
\hline $1.000 \mu \mathrm{L}$ & $1 / 800$ & 0 & 0 & 5 & $100 \%$ \\
$500 \mu \mathrm{L}$ & $1 / 1600$ & 0 & 0 & 6 & $100 \%$ \\
$200 \mu \mathrm{L}$ & $1 / 4000$ & 0 & 13 & 0 & $98 \%$ \\
$100 \mu \mathrm{L}$ & $1 / 8000$ & 0 & 16 & 0 & $98 \%$ \\
$50 \mu \mathrm{L}$ & $1 / 16000$ & 34 & 0 & 0 & $100 \%$ \\
$25 \mu \mathrm{L}$ & $1 / 32000$ & 31 & 0 & 0 & $100 \%$ \\
\hline
\end{tabular}

Fonte: Medeiros C. Z:; Ehrhardt A.; Cruz L.C. 2017.

Na tabela 3, a efetividade do produto foi comparada ao tempo em minutos (min), em que as larvas levaram para serem inibidas nas diluições apresentadas, indicando a porcentagem de mortalidade e a eficiência (E\%) do produto.
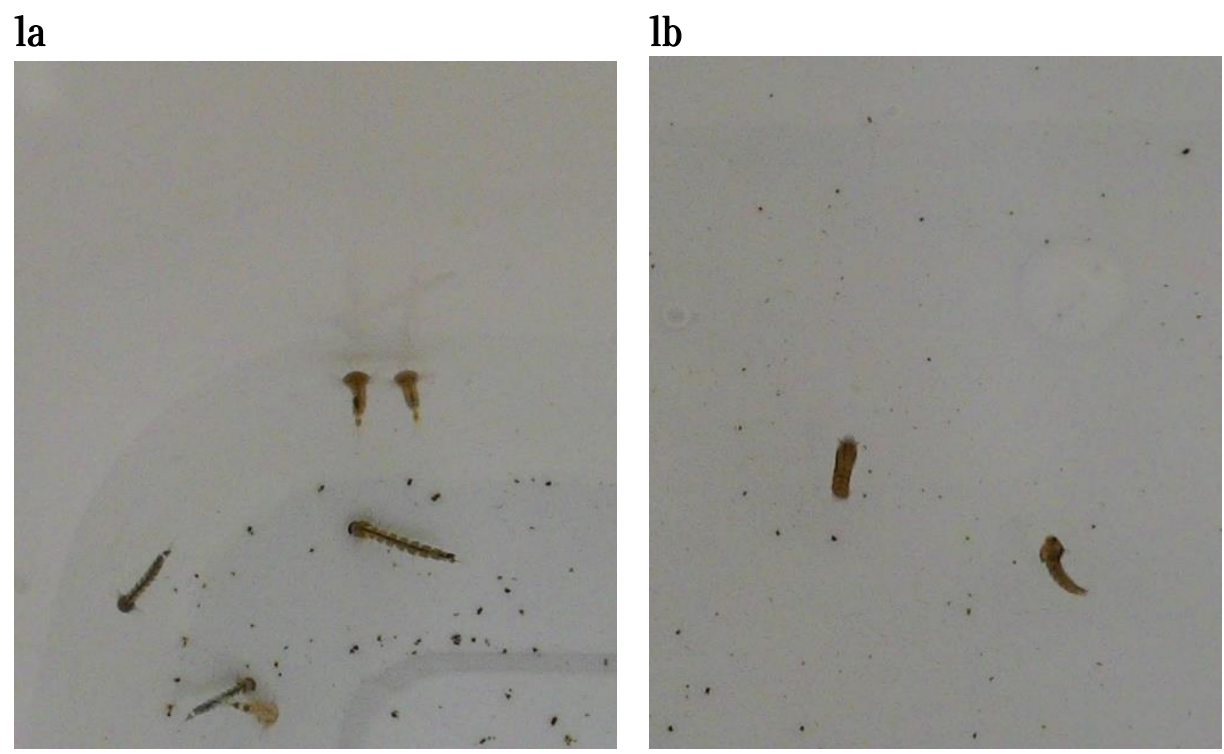

Figura 1. la - Culicídeos em fase larvária de criadouros em localidades de foco; lb - Culicídeos em fase pupa de criadouros em localidades de foco.

Fonte: Medeiros C. Z.; Ehrhardt A; Cruz L.C. 2017. 
A escolha de um inseticida deve ser realizada através da sua efetividade diante a espéciealvo susceptível e a segurança sobre o meio ambiente e aos seres vivos expostos a esses componentes. A OMS (Organização Mundial da Saúde), recomenda a utilização do grupo dos piretróides ao gênero Anopheles, por apresentar sensibilidade a esse grupo, sendo eficaz e seguro diante do efeito residual através de borrifação intradomicilar. Entre os indicados, pode ser citado, a DMT e a LCT, com residualidade estimada de dois a seis meses (SANTOS, et al., 2007).

Nos primeiros estudos de suscetibilidade ao A. aegypti à DMT na concentração de 1\%, aplicada em ultrabaixo volume (UBV), na dosagem de $0,8 \mathrm{~g} / \mathrm{Ha}$, na cidade de Goiânia, por Camargo et al. (1994) e Silva et al. (1994) apresentaram mortalidade de 94 a 98\% nos testes realizados. As fêmeas da espécie A. aegypti podem também apresentar uma redução da oviposição em superfícies tratadas com DMT (KONGMEE et al., 2004).

A LCT em superfícies vegetativas, como folhas, apresenta alta efetividade diante de mosquitos adultos das espécies Aedes albopictus e Culex quinquefasciatus em bioensaios no prazo de 24 horas, indicando um bom perfil de repelência (CILEK et al., 2008).

Cabe destacar que existe uma variação dos efeitos dos piretróides em diferentes condições, que incluem a aplicação em diferentes ambientes e superfícies relacionadas às residências, como temperatura e umidade dos locais, limpeza das paredes, iluminação e ventilação do local. Em estudos com a DMT, a mesma apresentou desempenho equivalente em todas as superfícies testadas. A redução do efeito foi gradual e regular, tendo o período de três meses nas superfícies de madeira e alvenaria. A LCT apresentou efetividade menos prolongada, não havendo diferença estatística entre as superfícies testadas e apontando após o segundo mês perda da atividade inseticida indiferentemente da superfície testada (SANTOS et al., 2007).

Em experimentos avaliando a eficácia de alfa-cipermetrina e LCT na prevenção da colonização de larvas de mosquitos em recipientes contendo água, foram realizadas duas diferentes aplicações em pneus de automóveis em desuso na cidade de Darwin, Austrália. Os pneus de controle foram colonizados por Aedes notoscriptus e Culex quinquefasciatus. 0 A. notoscriptus teve redução da propagacão em pneus tratados com alfa-cipermetrina até a $22^{\mathrm{a}}$ semana e não colonizou pneus tratados com LCT durante o teste de 24 semanas. Somente o $C$. quinquefasciatus conseguiu colonizar os pneus tratados com alfa-cipermetrina e lambdacialotrina entre $11^{\mathrm{a}}$ e $15^{\mathrm{a}}$ semana, respectivamente. Estes resultados indicam que ambos os inseticidas evitaram a colonização de A. notoscriptus por 20 semanas e demonstraram grande potencial de prevenção na reprodução em recipientes pela gênero Aedes, incluindo o A. aegypti e A. albopictus, sendo os principais maiores vetores da dengue (PETTIT et al., 2010). 
Com o intuito de novas estratégias para controle vetorial, o estudo de Silva et al. (2017), analisou durante o período de exposição de 24 horas, larvas de A. aegypti aos óleos essenciais de Hyptis suaveolens e Vanillosmopsis arbórea, os quais, verificaram maior mortalidade das larvas testadas, em comparação com outras plantas em estudo, adotando-as como biolarvicida. Todavia, o potencial tóxico de óleos essenciais e seus compostos contra o A. aegypti, pode variar conforme fatores intrínsecos e extrínsecos considerados em estudo.

Em um estudo realizado na Colômbia em 2013, foi avaliada a sensibilidade de populações naturais de $A$. aegypti na presença de inseticidas autorizados pelo programa de controle nacional, verificando que as populações estudas apresentaram sensibilidade a organofosforados, temephos, malathion e fenitrotion. 0 uso de organofosforados, como 0 malathion e o temephos foi implementado desde 1990, sendo os compostos químicos empregados por entidades estatais de saúde para o controle da dengue. Porém o uso do malation não obteve uma boa aceitação, devido ao odor e ação corrosiva em metais. Do contrário, o uso de temephos teve melhor acessão na comunidade, tendo uma ação categórica em larvas sem os efeitos adversos do malathion (ROLDAN et al., 2013).

De acordo com a Vigilância Epidemiológica do município e departamento de Saúde Pública do Rio Grande do Norte, no estudo de Nunes et al. (2016), foi relatado, que o uso do organofosforado Temephos, foi empregado durante a última década em grande escala em várias regiões no estado.

As MF0s (oxidase de função mista), são enzimas de detoxicação importantes na detecção de resistência a organofosoforados em diversos espécimes de vetores. No estudo de Nunes et al. (2016) constatou que essas enzimas apresentaram maior perfil de resistência entre três populações de Aedes, sendo avaliada como substancialmente alterada. Desse fato, sugere-se de que 0 uso de piretróides e organofosforados simultaneamente durante anos como estratégia de controle vetorial, poderia ser a causa da seleção de populações resistentes no município de estudo. Outro estudo também apontou resistência em populações selecionadas de A. aegypti na Tailândia com altos níveis aumentados de MF0 (PAEPORN et al., 2014). 
Tendo em vista a seleção de indivíduos resistentes, ocasionando diminuição da eficiência dos produtos utilizados para o controle vetorial, ressalta-se que o maior problema gerado através desse fator, é o alargamento do risco ambiental devido ao maior número de aplicações e aos diferentes mecanismos de atuação associados. Essa ação simultânea ocorre normalmente quando é empregado mais de um pesticida (CASTR0; QUEIR0Z, 2011).

A detecção da resistência e dos mecanismos envolvidos exploram as características de desvantagens adaptativas diante a insetos resistentes que apresentam menor aptidão que os suscetíveis quando não há pressão seletiva. Esse fator pode estar associado a menor fecundidade dos insetos, maior tempo de desenvolvimento, menor competitividade para 0 acasalamento e maior suscetibilidade aos oponentes naturais. Avaliando essas habilidades competitivas e a existência desse curso adaptativo em populações de A. aegypti, é o que norteiam os programas de manejo para análise da resistência quanto à desvantagem adaptativa ao inseto na ausência de pressão de seleção (DINIZ et al., 2014).

A prevenção acerca da seleção de indivíduos resistentes, é um dos principais fatores para monitoramento de vetores em regiões de grande manejo e dispersão simultânea de inseticidas. Essa verificação, auxilia nos programas de controle para não proliferação de espécies com potencial vetorial, do mesmo modo que certifica o uso adequado dos compostos empregados impedindo o surgimento de espécimes resistentes (CASTR0; QUEIROZ, 2011).

Com base nos surtos de doenças transmitidas pelo A. aegypti evidenciados atualmente, e seguindo as medidas de prevenção e controle adotados pelas entidades e órgãos públicos, a implementação de novas estratégias de combate ao vetor, torna-se essencial p a interrupção do ciclo biológico e repelir os mosquitos dos locais de postura (COELHO et al., 2017).

\section{CONSIDERAÇÕES FINAIS}

Ao serem avaliadas as larvas de culicídeos selvagens expostas a aplicação do produto com ação larvicida na combinação dos piretróides DMT e LCT, observou-se que a exposição diante as diferentes diluições realizadas, apresentaram inibição total da motilidade das larvas, indicando assim, efetividade nos experimentos realizados mesmo em concentrações menores do que o recomendado pelo fabricante.

Este estudo revela a necessidade do acompanhamento em pesquisas pelas vigilâncias regionais, visando o monitoramento e desenvolvimento de novas tecnologias e implementação 
Aedes Aegypti: Estudo Piloto sobre a Efetividade de Deltametrina e Lambda-Cialotrina em

Culicídeos com Capacidade Vetorial

de estratégias diligentes para não proliferação de mosquitos vetores como o A. aegypti em áreas de risco.

\section{AGRADECIMENTOS}

Gostaríamos de agradecer a empresa Landrin Indústria e Comércio de Inseticidas LTDA, pela disponibilização dos princípios ativos testados em nosso estudo.

\section{REFERÊNCIAS BIBLIOGRÁFICAS}

BRAGA I. A.; VALLE D. 2007. Aedes aegypti: histórico do controle no Brasil. [Epidemiologia e Serviços de Saúde 2007; 16(2): 113 - 118].

BRASIL. Ministério da Saúde. Regulamento técnico Deltametrina e Lambda-cialtrona. Consulta Pública ${ }^{0} 35$, de 22 de maio de 2003.

BRASIL. Ministério da Saúde. Boletim Epidemiológico Secretaria de Vigilância em Saúde - Brasil, Volume 48 № 29 $-2017$.

BRASIL. Ministério da Saúde. Dengue, instruções para pessoal de combate ao vetor - manual de normas técnicas. 2001. p. 75.

CAMARG0, M. de F. et al. Diversidade e flutuação da entomofauna de Diptera Nematocera, na cidade de Goiânia. Rev. Pai. Trop., 25:321,1994.

CASTR0, E. A.; QUEIROZ, P. R. 0 vírus da dengue no brasil e as medidas de prevenção, controle e erradicação. [s.d.]. Disponível em: <http://www.cpgls.ucg.br/8mostra/Artigos/SAUDE E BIOLOGICAS/0 VIRUS DA DENGUE N0 BRASIL E AS MEDIDAS DE PREVENCA0, CONTROLE E ERRADICACA0.pdf`. Acesso em: 10 ago. 2017.

CILEK J. E. et al. Residual effectiveness of three pyrethroids on vegetation against adult Aedes albopictus and Culex quinquefasciatus in screened field cages. J Am. Mosq. Control Assoc. 24:263-269, 2008.

COELHO W. M. D. AlTERnATIVAS NO CONTROLE QUíMICO DO Aedes aegypti E Culex quinquefasciatus: ESTUDO PRELIMINAR SOBRE A EFICÁCIA DE PARASITICIDAS DE USO EXTERNO CONTRA LARVAS DE CULICÍDEOS. Colloquium Agrariae, v. 13, n.l, Jan-Abr. 2017, p.01-06. D0I: 10.5747/ca.2017.vl3.nl.al41

DINIZ, M. M. C. De S. L. et al. Resistance of aedes aegypti to temephos and adaptive disadvantages. Revista de saude publica, 2014. v. 48 , n. 5, p. $775-782$.

GUZMAN, M. G; E. HARRIS. Dengue. September 14, 2014 Disponível em: http://dx.doi.org/10.1016/S01406736(14)60572-9. Acesso em: 10 ago. 2017.

INSTITUT0 OSWALD0 CRUZ (IOC/FIOCRUZ). Ministério da Saúde. Curiosidade sobre o Aedes aegypti. Manguinhos, RJ - Brasil, 2012.

INVEST, J. F.; LUCAS, J. R. Pyriproxyfen as a mosquito larvicide. Procedings of the sixth international conference on urban pests, 2008. n. Who 2003, p. 239-245.

KONGMEE M. et al. Behavioral responses of Aedes aegypti (Diptera: Culicidae) exposed to deltamethrin and possible implications for disease control. Jornal Med Entomologia 41:1055-1063, 2004. 
MELÓ, M. E. B. DE. Investigação da genoticidade de larvicidas biologicos e sinteticos para o controle de Aedes aegypti. Universidade de Pernambuco. Centro de Ciências da saúde. Departamento de ciências farmacêuticas. Recife - PE / 2009.

NUNES et al. Caracterização de perfis enzimáticos de cepas do Aedes aegypti do Estado do Rio Grande do Norte, Brasil. Faculdade de Ciências da Saúde, Universidade do Estado do Rio Grande do Norte, Mossoró, Brasil. Ciência \& Saúde Coletiva, 21(1):285-292, 2016.

PAEPORN P. et al. Biochemical detection of pyrethroid resistance mechanism in Aedes aegypti in Ratchaburi province, Thailand. Trop. Biomed. 2004; 2(21):145-151.

PETTIT W. J. et al. Efficacy of Alpha-Cypermethrin and Lambda-Cyhalothrin Applications to Prevent Aedes Breeding in Tires. Journal of the American Mosquito Control Association, 26(4):387-397, 2010. Disponível em: http://www.bioone.org/doi/full/10.2987/09-5962.1. Acesso em: 10 ago. 2017.

PONTE, H. J. et al. Evaluation of pain manifestations in patients admitted to a reference hospital with presumptive dengue fever diagnosis. Revista dor, 2011. v. 12, n. 2, p. 104-107. Disponível em: $\langle$ http://www.scielo.br/scielo.php?script=sci_arttext\&pid=S1806-001320110002000058lng=en\&nrm=iso\&tlng=pt $\rangle$. ]. Acesso em: 10 ago. 2017.

ROLDAN S. A. et al. Estado de la sensibilidad a los insecticidas de uso en salud pública en poblaciones naturales de Aedes aegypti (Diptera: Culicidae) del departamento de Casanare, Colombia. Facultad de Agronomía, Universidad Nacional de Colombia, Bogotá, D.C., Colombia. Laboratorio de Entomología, Dirección de Red Nacional de Laboratorios, Instituto Nacional de Salud, Bogotá, D.C., Colombia 2013:33:446-58. http://dx.doi.org/10.7705/biomedica.v33i3.1534. Acesso em: 10 ago. 2017.

SANTOS R. L . C. dos. et al. Avaliação do efeito residual de piretróides sobre anofelinos da Amazônia brasileira. Revista Saúde Pública 2007;41(2):276-83.

SANTOS, M. A. T. dos; AREAS, M. A.; REYES, G. Piretróides - Uma visão geral. Departamento de Ciência de Alimentos - Faculdade de Engenharia de Alimentos - Universidade Estadual de Campinas, UNICAMP, Campinas, SP,Brasil. Araraquara v.18, n.3, p. 339-349, jul./set. 2007.

SILVA, T. I. da. et al. Efeito larvicida de óleos essenciais de plantas medicinais sobre larvas de Aedes aegypti L. (Diptera:Culicidae). Revista Verde de Agroecologia e Desenvolvimento Sustentável. V.12, No 2, p. 256-260, 2017 Pombal, PB, Grupo Verde de Agroecologia e Abelhas.

STEFANI G. P. et al. Repelentes de insetos: recomendações para uso em crianças. Universidade de Alergia e Imunologia do Instituto da Criança do Hospital de Clinicas da Faculdade de Medicina da Universidade de São Paulo, SP, Brasil. Ver Paul Pediatr. 2009; 27 (1): 81-9.

ZARA, A. L. de S. A. Estratégias de controle do Aedes aegyptí. uma revisão. Epidemiol. Serv. Saúde. Brasília, 25(2):391404, abr-jun 2016. 